\title{
Long-Term Survival with Growth Hormone Replacement after Liver Transplantation of Pediatric Nonalcoholic Steatohepatitis Complicating Acquired Hypopituitarism
}

\author{
Atsushi Fujio, ${ }^{1}$ Naoki Kawagishi, ${ }^{1}$ Taketora Echizenya, ${ }^{2}$ Kazuaki Tokodai, ${ }^{1}$ \\ Chikashi Nakanishi, ${ }^{1}$ Shigehito Miyagi, ${ }^{1}$ Kazushige Sato, ${ }^{1}$ Keisei Fujimori ${ }^{1}$ and \\ Noriaki Ohuchi ${ }^{1}$ \\ ${ }^{1}$ Division of Transplantation, Reconstruction, and Endoscopic Surgery, Tohoku University Hospital, Sendai, \\ Miyagi, Japan \\ ${ }^{2}$ Department of Pediatrics, Iwate Prefectural Chubu Hospital, Kitakami, Iwate, Japan
}

\begin{abstract}
Nonalcoholic steatohepatitis (NASH) is the most severe form of nonalcoholic fatty liver disease (NAFLD). In adult patients, liver transplantation (LT) is the treatment of choice for end-stage liver disease secondary to NASH. However, little information is available regarding outcomes of LT in pediatric patients with NASH. We describe here a pediatric patient with NASH associated with hypopituitarism who underwent living donor liver transplantation (LDLT). An 11-year-old boy was diagnosed with a pituitary tumor, which was removed by trans-interhemispheric approach following bifrontal craniotomy. Histopathological examination revealed a mature teratoma. Eighteen months later, magnetic resonance imaging showed recurrence of the pituitary tumor, which was found to be a germinoma. He underwent 3 months of chemoradiotherapy, with a complete response. He gradually became obese, with elevated transaminase levels. At age 15 years, he developed fatigue and dyspnea and was found to have liver cirrhosis secondary to NASH with severe hepatopulmonary syndrome. He underwent LDLT using a right liver graft from his mother. Twelve months later, abdominal computed tomography showed recurrence of NAFLD. Five years after the LDLT, transaminases were slightly elevated. Growth hormone replacement therapy was started, reducing transaminase levels to their normal ranges. Ten years after LDLT, fatty liver remains stable, although his body mass index has not been reduced. Growth hormone replacement therapy may be effective in graft maintenance. This is the first case report of a patient with maintained stable liver function 10 years after LDLT for pediatric NASH.
\end{abstract}

Keywords: child; growth hormone; hypopituitarism; liver transplantation; nonalcoholic steatohepatitis Tohoku J. Exp. Med., 2015 January, 235 (1), 61-67. (C) 2015 Tohoku University Medical Press

\section{Introduction}

Nonalcoholic steatohepatitis (NASH), the most severe form of nonalcoholic fatty liver disease (NAFLD) (Ludwig et al. 1980), is characterized histologically by steatohepatitis, hepatocellular ballooning, and inflammation in the absence of alcohol consumption or other causes of steatosis (Aly and Kleiner 2011). Progressive fibrosis occurs in $10-15 \%$ of patients with NASH and cirrhosis may develop in $15-25 \%$ of these patients (Farrell and Larter 2006). Liver transplantation (LT) is the treatment of choice for end-stage liver disease secondary to NASH. Because of the epidemic of metabolic syndrome, the proportion of adult patients with NASH undergoing LT has increased markedly, and appears to be continuing to increase (Afzali et al. 2012). Recently, NAFLD and NASH have also been recognized in children (Giorgio et al. 2013). At present, however, little information is available regarding outcomes of LT in pediatric NASH. Few case reports have documented their posttransplant prognosis, but none has analyzed long-term survival (Jonas et al. 2005; Jankowska et al. 2007).

We describe here a boy who developed NASH after surgical removal of a pituitary tumor. This patient initially developed hypopituitarism, followed by NASH and severe hepatopulmonary syndrome (HPS), and underwent living donor liver transplantation (LDLT) at age 15 years. He was followed-up for 10 years. This is a rare case report showing the maintenance of stable liver function for 10 years following LDLT for pediatric NASH.

Case Presentation

An 11-year-old boy was admitted to a local hospital

Received September 11, 2014; revised and accepted January 5, 2015. Published online January 17, 2015; doi: 10.1620/tjem.235.61. Correspondence: Atsushi Fujio, M.D., Ph.D., Division of Transplantation, Reconstruction, and Endoscopic Surgery, Tohoku University Hospital, 1-1 Seiryo-machi, Aoba-ku, Sendai, Miyagi 980-0872, Japan.

e-mail: wisteriatail@med.tohoku.ac.jp 
(A)

(C)

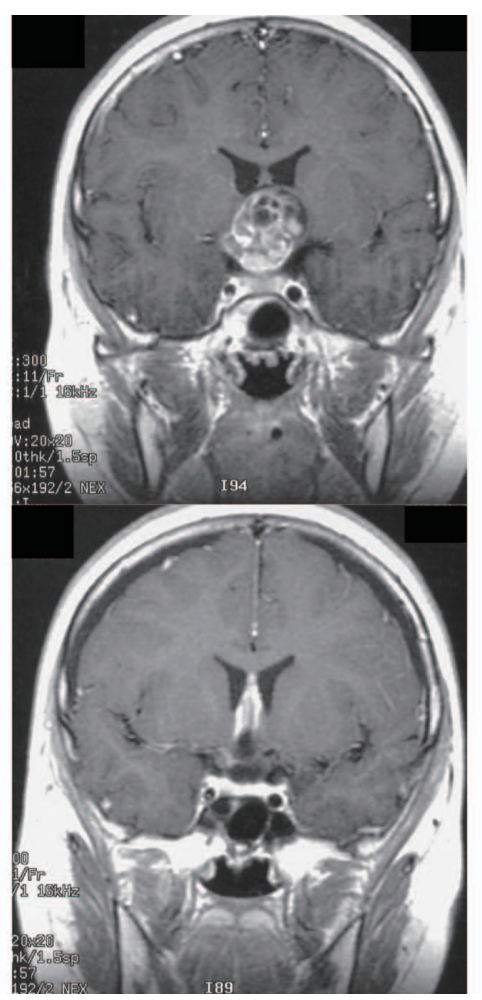

(B)

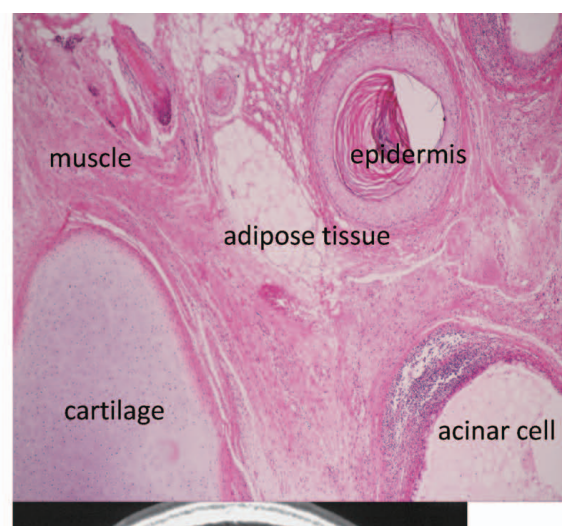

(D)

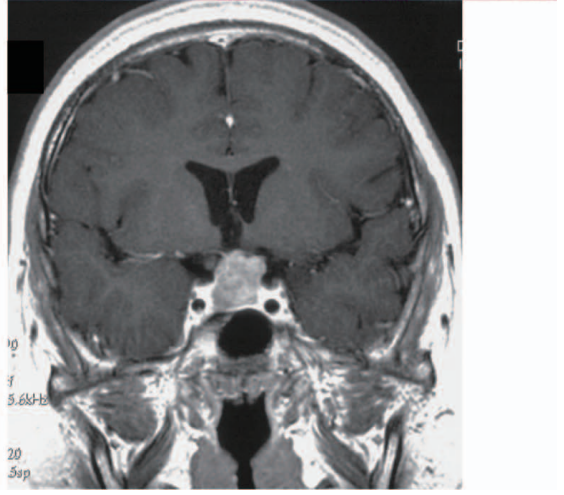

Fig. 1. MRI images and a histological picture of the pituitary tumor.

The primary pituitary tumor was mature teratoma, and the recurrence tumor was germinoma.

$(\mathrm{A}, \mathrm{B})$ The primary pituitary tumor on the supurasellar area contained high and low signal intensity areas and was heterogeneously enhanced by gadolinium. The specimen was contained epidermis, muscle, and acinar cell, which originate from ectodermal, mesodermal and endodermal cells, respectively. (C) Post operative MRI showed no remnant of the tumor. (D) MRI showed that the recurrent sellar tumor was homogeneously enhanced by gadolinium.

because of polyposia. Magnetic resonance imaging (MRI) showed intracranial hypertension secondary to a pituitary tumor, accompanied by headache and nausea. The tumor contained high and low signal intensity areas and was heterogeneously enhanced by gadolinium (Fig. 1A). It was removed by trans-interhemispheric approach following bifrontal craniotomy, with histopathological examination showing that it was a mature teratoma (Fig. 1B). Endocrinological data revealed growth hormone (GH) 0.07 $\mathrm{ng} / \mathrm{mL}$; insulin-like growth factor-1 (IGF-1) $110 \mathrm{ng} / \mathrm{mL}$; adrenocorticotropic hormone $6 \mathrm{pg} / \mathrm{mL}$; free triiodothyronine $1.12 \mathrm{pg} / \mathrm{mL}$; free thyroxin $0.69 \mathrm{ng} / \mathrm{mL}$; thyroid stimulating hormone $0.152 \mu \mathrm{IU} / \mathrm{mL}$ and cortisol $14.3 \mu \mathrm{g} / \mathrm{dL}$. After the operation, he was treated for hypopituitarism and hypothalamic dysfunction with intranasal desmopressin, glucocorticoid, and thyroxine replacement therapy. We also performed GH provocative tests at this time. His GH response was extremely low (peak GH: clonidine test, 0.1 $\mathrm{ng} / \mathrm{ml}$; arginine test, $0.27 \mathrm{ng} / \mathrm{ml}$ ). Therefore, he received $1.33 \mathrm{mg}$ per day GH replacement therapy for 12 months.

Eighteen months after the operation, MRI showed that the recurrence of a pituitary tumor was homogeneously enhanced by gadolinium different from primary tumor. Thereafter, radiation therapy with 9.6 Gy was performed, and the tumor was reduced markedly, found to be a germinoma (Fig. 1C, D). He underwent 3 months of chemora- diotherapy, resulting in a complete response. During follow-up, laboratory tests revealed lower platelet counts and slightly elevated serum transaminase and total bilirubin levels. Abdominal computed tomography (CT) scans showed evidence of fatty liver. His body mass index (BMI) increased yearly owing to hyperphagia.

At age 15 years, he developed fatigue and dyspnea. Physical examination showed that he was obese, with a weight of $95 \mathrm{~kg}$ and a BMI of $29.3 \mathrm{~kg} / \mathrm{m}^{2}$, with hypoxemia $\left(\mathrm{PaO}_{2} 51.3 \mathrm{mmHg}, \mathrm{O}_{2} 3 \mathrm{l} / \mathrm{min}\right)$ and peripheral cyanosis. An abdominal CT scan showed hepatosplenomegaly. A liver biopsy revealed liver cirrhosis secondary to NASH (Fig. 2). NASH activity score (NAS) was 6. Matteoni's classification was type 3. Brunt's grading and staging were grade 3 and stage 4 , respectively. Lung perfusion scintigraphy with $\mathrm{Tc}^{99 \mathrm{~m}}$-macroaggregated albumin showed intrapulmonary shunting without pulmonary hypertension, with a shunt ratio calculated from the perfusion scintigram of $57.5 \%$. Laboratory data revealed total bilirubin $2.9 \mathrm{mg} / \mathrm{dL}$; direct bilirubin $1.0 \mathrm{mg} / \mathrm{dL}$; alkaline phosphatase $391 \mathrm{U} / \mathrm{L}$; gammaglutamic transpeptidase $43 \mathrm{U} / \mathrm{L}$; aspartate aminotransferase $22 \mathrm{U} / \mathrm{L}$; alanine aminotransferase $16 \mathrm{U} / \mathrm{L}$; albumin $3.9 \mathrm{~g} /$ dL; white blood cell count $6,200 / \mu \mathrm{L}$; hemoglobin $13.7 \mathrm{~g} /$ $\mathrm{dL}$; platelet count $42,000 / \mu \mathrm{L}$; and prothrombin time international normalized ratio; 1.45 . His fasting glucose level was normal $(72 \mathrm{mg} / \mathrm{dL})$, but his insulin level $(22.8 \mu \mathrm{U} / \mathrm{mL})$ 
(A)

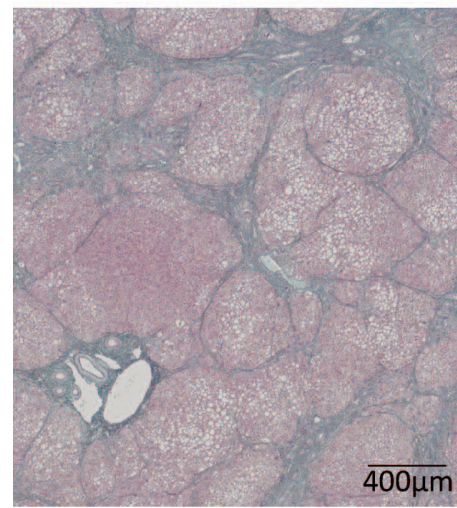

(B)

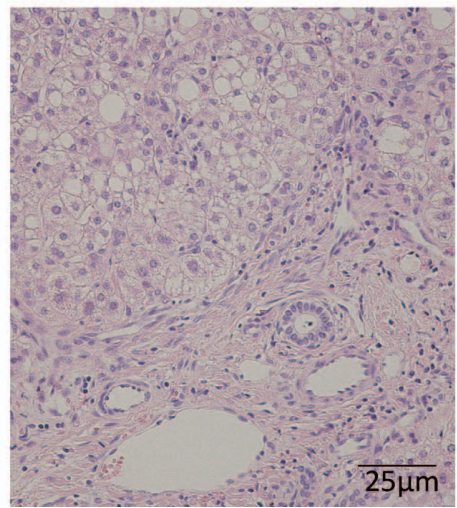

(C)

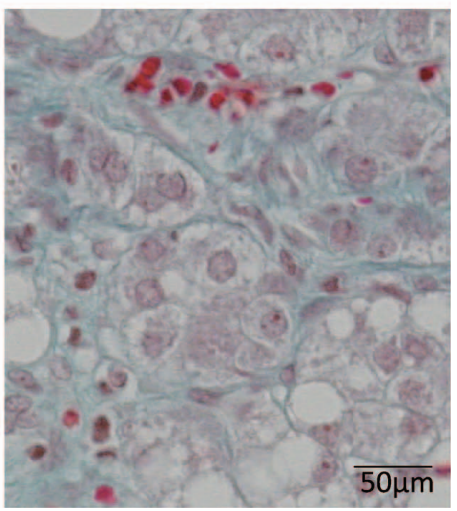

Fig. 2. Liver biopsy specimen showing severe steatosis and fibrosis before LT.

The specimen was characterized by diffuse steatosis with an inflammatory infiltrate and hepatocyte ballooning, as well as by perisinusoidal and pericellular fibrosis with macrovesicular steatosis, followed by bridging fibrosis. (A) ElasticaMasson staining; original magnification, $\times 20$. (B) Hematoxylin-eosin staining; original magnification, $\times 200$. (C) Elastica-Masson staining; original magnification, $\times 400$.

and homeostasis model assessment-insulin resistance (HOMA-IR) (4.05) were high. He was negative for viral infections, including hepatitis $\mathrm{B}$, hepatitis $\mathrm{C}$, and cytomegalovirus, as well as for metabolic diseases. He had no ascites and encephalopathy, correlating with a Child-Pugh Score of 8 (class B) and a model for end-stage liver disease (MELD) score of 15 . Based on these findings, he was diagnosed with liver cirrhosis secondary to NASH with severe HPS and referred to our hospital for LDLT.

LDLT was performed using a right liver graft from his mother. This program was approved by the ethics committee of Tohoku University Hospital, and the patients provided written informed consent for all procedures and therapies. The procedure took 17 hours and 53 minutes. The graft weight was $595 \mathrm{~g}$ and the ratio of graft volume to standard liver volume was $43 \%$. Total and warm ischemic times were $253 \mathrm{~min}$ and $91 \mathrm{~min}$, respectively. The explanted graft weight was $1,621 \mathrm{~g}$. Histopathological examination revealed steatosis, hepatocellular ballooning, and lobular inflammation. It was diagnosed with liver cirrhosis secondary to NASH. Initial immunosuppression treatment consisted of tacrolimus, methylprednisolone, and basiliximab following our own protocol. The graft function was excellent on the postoperative course, and extubation could be performed on postoperative day (POD) 11. After extubation, it took a lot of days to control diabetes insipidus. Laboratory data such as urine specific gravity, urine and serum osmolality and serum electrolytes were required to determine the amount of intranasal desmopressin for management of postoperative fluid intake and output. After his diabetes insipidus and several treatments for cytomegalovirus infection were controlled, he was discharged from the hospital on POD 147. At this time, the shunt ratio calculated from the perfusion scintigram was $25.4 \%$. And his arterial blood gas analysis was also improved $\left(\mathrm{PaO}_{2} 68.6\right.$ mmHg, room air) (Fig. 3).
Twelve months after LDLT, follow-up laboratory tests revealed lower platelet counts and prolongation of prothrombin time. CT scan showed stenosis of the portal vein anastomosis, which was confirmed as portography. Although balloon dilation was successful, laboratory results did not improve, and a CT scan detected slightly fatty liver, suggesting recurrence of NAFLD in the graft. Five years after LDLT, transaminase levels were remained slightly elevated. Endocrinological data revealed GH $0.04 \mathrm{ng} / \mathrm{mL}$ and IGF-1 $41 \mathrm{ng} / \mathrm{mL}$. Therefore he was diagnosed with adult GH deficiency. He was started $0.2 \mathrm{mg}$ per day GH replacement therapy, resulting in decreased transaminase levels to their normal ranges. Moreover, fatty liver detected by the CT scan was also improved (Fig. 4). Endocrinological data revealed GH $0.12 \mathrm{ng} / \mathrm{mL}$; IGF-1 $71 \mathrm{ng} / \mathrm{mL}$; Luteinizing hormone $<0.1 \mathrm{mIU} / \mathrm{mL}$; Follicle stimulating hormone 0.06 $\mathrm{mIU} / \mathrm{mL}$ and testosterone $<0.03 \mathrm{ng} / \mathrm{mL}$. Six years after LDLT, type 2 diabetes mellitus was diagnosed, and the patient was started on metformin and glimepiride. Until now, he repeated dehydration caused by diabetes insipidus many times. And he was required hospitalization each time. Currently, 10 years after LDLT, his fatty liver remains stable, with the portal vein being patent by a CT scan. Although his BMI remains high $\left(31.0 \mathrm{~kg} / \mathrm{m}^{2}\right)$, his liver functions remain stable without any signs of brain tumor recurrence (Fig. 5).

\section{Discussion}

NASH is the progressive form of NAFLD, which has recently emerged as the leading cause of chronic liver disease in children (Giorgio et al. 2013). It has been estimated that $15-20 \%$ of patients with NAFLD have NASH, a condition associated with central obesity, type 2 diabetes mellitus, and insulin resistance, the main features of the metabolic syndrome (Nanda 2004). Patients with hypothalamic disorders and hypopituitarism, who are at risk of excessive 


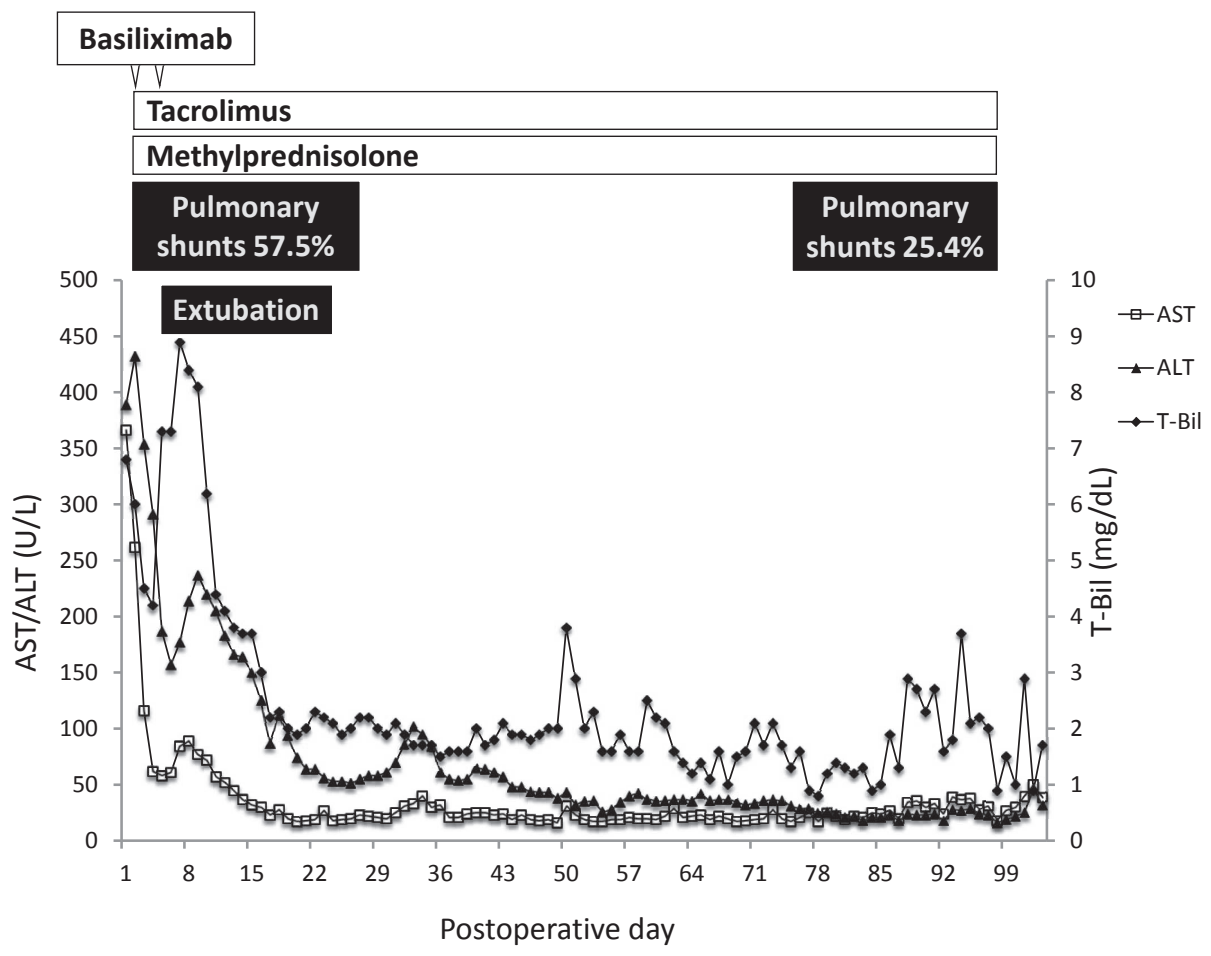

Fig. 3. Clinical course in the patient for postoperative days.

Initial immunosuppression treatment consisted of tacrolimus, methylprednisolone, and basiliximab. The shunt ratio calculated from the perfusion scintigram was improved after LT. AST, aspartate aminotransferase; ALT, alanine aminotransferase; T-Bil, total bilirubin.

(A)

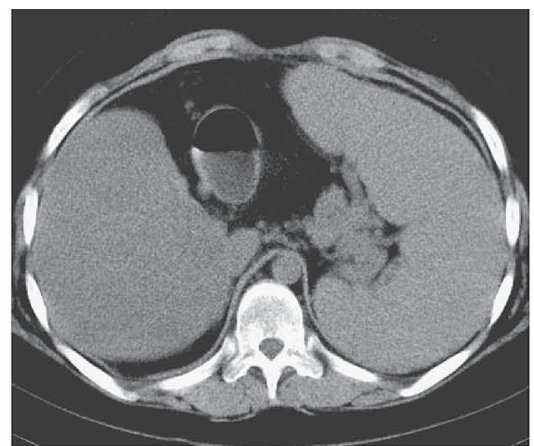

(C)

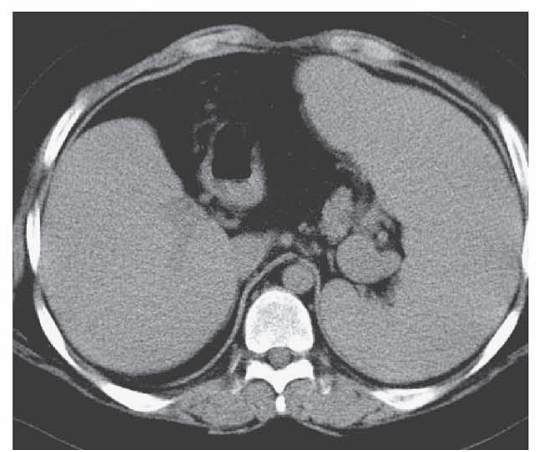

(B)

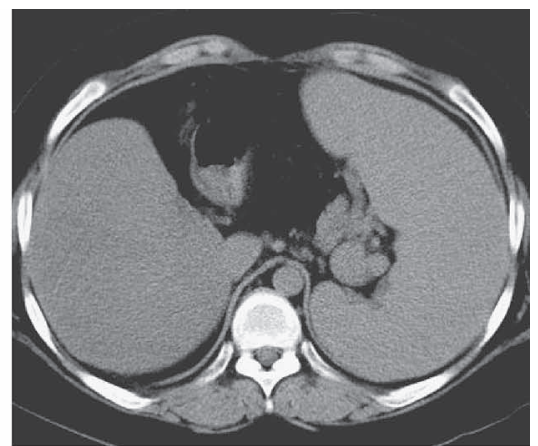

Fig. 4. Follow-up findings.

(A) At 18 months after LDLT, slightly fatty liver was detected, with a mean CT level of 33 HU. (B) At 48 months after LDLT, stable fatty liver was detected, with a mean CT level of 32 HU. (C) At 72 months after LDLT, fatty liver has improved after the growth hormone replacement therapy, with a mean CT level of $50 \mathrm{HU}$. 
(A)

\begin{tabular}{|l|l|l|l|l}
\hline Chemoradiotherapy & GDLT replacement therapy \\
\hline
\end{tabular}

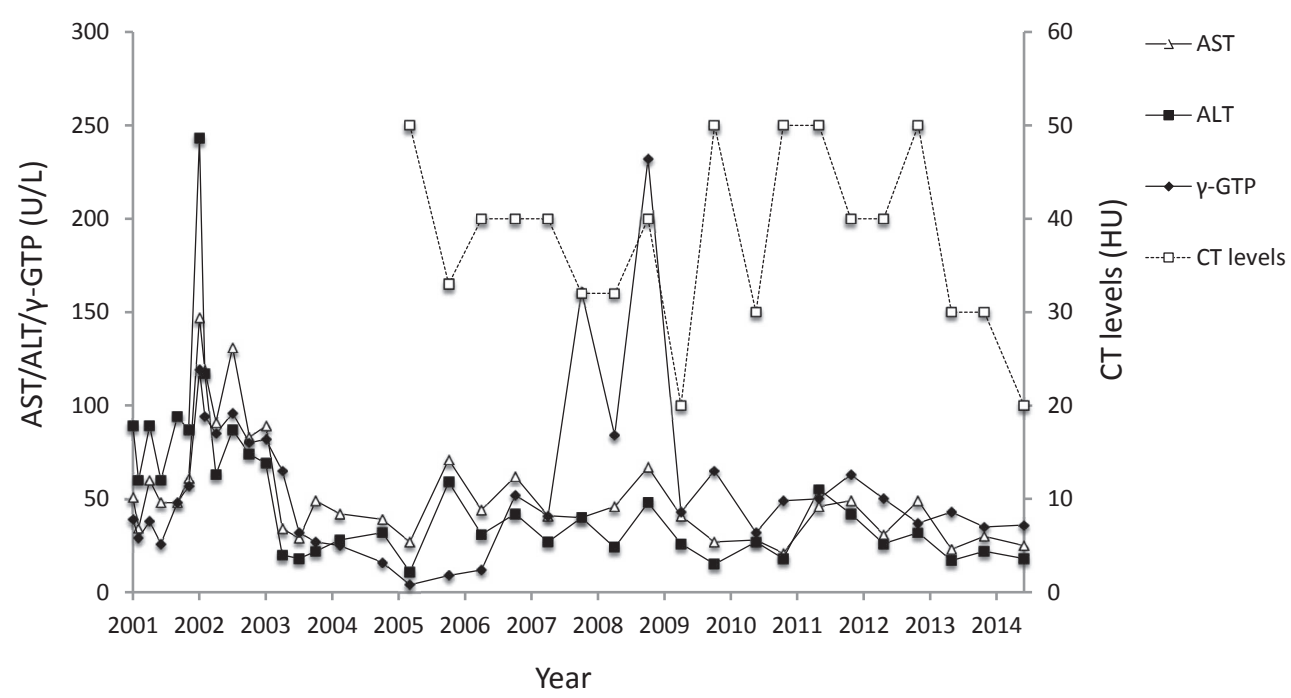

(B)

\begin{tabular}{|l|l|}
\hline Chemoradiotherapy & LDLT \\
\hline
\end{tabular}

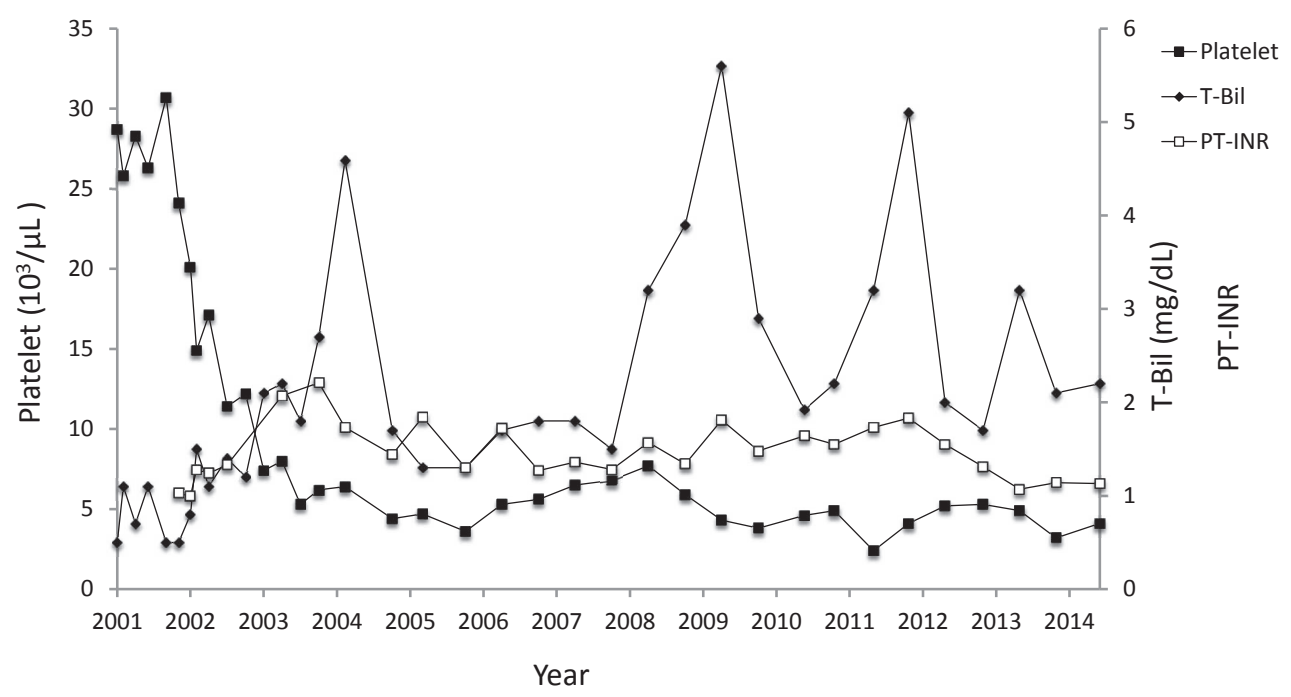

Fig. 5. Clinical course in the patient for 13 years.

(A) The data of transaminases, $\gamma$-GTP and CT levels in the clinical course. (B) The data of platelet, total bilirubin and PT-INR in the clinical course. Chemoradiotherapy, LDLT, and GH replacement therapy were performed in 2002, 2004, and 2009, respectively. AST, aspartate aminotransferase; ALT, alanine aminotransferase; T-Bil, total bilirubin; LDLT, living donor liver transplantation; GH, growth hormone; $\gamma$-GTP, gamma-glutamic transpeptidase; PT-INR, prothrombin time international normalized ratio.

weight gain, impaired glucose tolerance, and hyperphagia, may also have NAFLD and NASH (Adams et al. 2004; Nakajima et al. 2005). NAFLD and NASH accompanying hypopituitarism are likely caused by GH deficiency and obesity (Rosen and Bengtsson 1990; Nakajima et al. 2005). Our patient experienced hypopituitarism and hypothalamic dysfunction following the removal of his pituitary tumor. Hypothalamic dysfunction may result in central obesity owing to hyperphagia. Central obesity from hyperphagia and/or GH deficiency from hypopituitarism are thought to be associated primarily with fatty infiltration of the liver, resulting in NAFLD and NASH. NASH may also be 
caused by corticosteroid replacement, a known cause of liver steatosis (Dourakis et al. 2002). However, the dose of corticosteroid administered to our patient was physiologic, replacing absent endogenous corticoids. This dosage was unlikely to have a significant role in the development and progression of NASH in our patient.

LT in NASH is increasing in parallel with the increased rates of obesity and diabetes mellitus. The posttransplant prognosis of adults with NASH is excellent, comparable to that observed in patients with other liver diseases (Afzali et al. 2012). LT, however, is rarely indicated for pediatric NASH. A 20-year follow-up of 66 children with NAFLD found that only two underwent LT for cirrhosis secondary to NASH with HPS (Feldstein et al. 2009). NASH recurred in these two patients 9 months and 6 weeks after LT, respectively. The latter patient underwent retransplantation, but died from multiple organ failure 8 years after the first LT.

Several studies have assessed outcomes of LT in patients with NAFLD and NASH accompanied by hypopituitarism and hypothalamic dysfunction. In one report, 21 patients were diagnosed with NAFLD 6.4 years after the diagnosis of pituitary and hypothalamic dysfunction (Adams et al. 2004). Of the 10 patients biopsied, six had cirrhosis, two had simple steatosis, and two had NASH with fibrosis requiring LT. Two other case reports described patients with cirrhosis secondary to NASH with hypopituitarism associated with HPS; both of these patients were diagnosed with recurrent NASH within 6 months after LT (Jonas et al. 2005; Jankowska et al. 2007). Even with careful monitoring and treatment of endocrine abnormalities, NAFLD and NASH recur in most of these patients after LT, with some of these patients requiring re-transplantation. In our patient, the diagnosis of cirrhosis secondary to NASH was confirmed by liver biopsy 4 years after surgical removal of the pituitary tumor. Interestingly, HPS was the main indication for LT in our patient. HPS may be more common in cirrhosis associated with hypopituitarism than in other types of cirrhosis (Jonas et al. 2005). The number of cases is too small to be statistically significant. However, in light of this tendency, this association should be suspected. The pathogenesis of HPS has not been identified. Most patients with HPS have cirrhosis and portal hypertension. Portal hypertension causes impairment in the perfusion of the bowel and increases the enteral translocation of Gram-negative bacteria and endotoxins. This stimulates the release of vasoactive mediators, such as tumor necrosis factor-alpha (TNF- $\alpha$ ) (Tumgor 2014). On the other hand, patients with adult GH deficiency have a high cardiovascular risk in relation to an increase of TNF- $\alpha$, which is also known as inflammation biomarker (Gonzalez-Duarte et al. 2012). Therefore, the pathological condition of HPS and adult GH hormone deficiency may be similar to an increase of TNF- $\alpha$. If confirmed by further research, this association may provide a key to the interrelated pathogenesis of these conditions and indications for LT in pediatric NASH.
Liver biopsy currently remains the gold standard for the diagnosis of NASH, but it has several important limitations (Younossi 2008; Tiniakos 2010). First, liver biopsy is associated with potentially severe complications. Second, liver biopsy can be associated with a sampling error. A liver biopsy could not be obtained from our patient after LT. One of the reasons was that his serum transaminase levels were sometimes elevated due to dehydration, but improved by intravenous rehydration each time. The other was his lower platelet counts and prolonged prothrombin time. $\mathrm{He}$ was followed closely by laboratory examinations, ultrasound, and CT. Although these noninvasive methods can detect hepatic steatosis, but the recurrence of NASH could not be assessed. Metabolic syndrome, including obesity and type 2 diabetes mellitus, plays an essential role in the recurrence of NASH after LT (El Atrache et al. 2012). Recurrence of NASH has not been observed in patients with significantly reduced weight after LT (Koneru and Dikdan 2002). A recent randomized study showed that a $7 \%$ weight reduction in patients with NASH significantly improved liver histology (Promrat et al. 2010). Our patient, however, showed an increase in BMI over 10 years, emphasizing the importance of weight reduction in our patient through lifestyle interventions.

Although his BMI has not been reduced, the liver function of our patient has remained stable for 10 years after LT. This may be owing in part to treatment with metformin, an agent shown effective for pediatric NAFLD and NASH (Schwimmer et al. 2005; Jankowska et al. 2007). Stable liver function in our patient may also be because of GH replacement therapy. Progression to NASH can be explained by the "two hit" hypothesis (Giorgio et al. 2013). The first hit consists of the intrahepatic accumulation of fatty acids within hepatocytes, which is closely associated with insulin resistance. The second hit consists of the increased oxidative stress, cytokine induction, and inflammation. This pathological cascade is similar to that of adult GH deficiency. GH replacement therapy for 6-12 months was shown to significantly reduce transaminase levels in 11 patients with NAFLD and to improve steatosis and fibrosis in six patients with NASH (Nishizawa et al. 2012a). It has been known that the liver is one of the important target tissues of GH (Takahashi 2012). GH improves visceral fat and triglyceride accumulation in hepatocytes (Takahashi et al. 2007). It also enhances the clearance of low-density protein (LDL) by activating the expression of hepatic LDL receptors. GH acts directly and indirectly through IGF-1, which is derived predominantly from hepatocytes. IGF-1 also reduces insulin resistance and oxidative stress. Moreover it improves mitochondrial function and may prevent the progression of fibrosis (Nishizawa et al. 2012b). Therefore, it is speculated that these GH and IGF-1 effects in the liver are directly attribute to the GH replacement therapy. In fact, our patient improved not only GH levels but also IGF-1 levels by GH replacement therapy. However, insufficient data are currently available to deter- 
mine whether GH replacement therapy should be a standard treatment for NASH. The relatively good response in our patient, as indicated by decreased transaminase levels, provides further evidence for the beneficial effect of GH replacement therapy in patients with NASH.

In conclusion, there have been few report of long-term survival following LT in pediatric NASH. This is a rare case report showing that stable liver function was maintained for 10 years after LDLT for pediatric NASH. GH replacement therapy may be effective in graft maintenance. Therefore additional reports of long-term survival following LT in pediatric NASH are needed.

\section{Acknowledgments}

The authors thank Dr. Takaaki Beppu (Iwate Medical University) for the patient data.

\section{Conflict of Interest}

The authors declare no conflict of interest.

\section{References}

Adams, L.A., Feldstein, A., Lindor, K.D. \& Angulo, P. (2004) Nonalcoholic fatty liver disease among patients with hypothalamic and pituitary dysfunction. Hepatology, 39, 909-914.

Afzali, A., Berry, K. \& Ioannou, G.N. (2012) Excellent posttransplant survival for patients with nonalcoholic steatohepatitis in the United States. Liver Transpl., 18, 29-37.

Aly, F.Z. \& Kleiner, D.E. (2011) Update on fatty liver disease and steatohepatitis. Adv. Anat. Pathol., 18, 294-300.

Dourakis, S.P., Sevastianos, V.A. \& Kaliopi, P. (2002) Acute severe steatohepatitis related to prednisolone therapy. Am. J. Gastroenterol., 97, 1074-1075.

El Atrache, M.M., Abouljoud, M.S., Divine, G., Yoshida, A., Kim, D.Y., Kazimi, M.M., Moonka, D., Huang, M.A. \& Brown, K. (2012) Recurrence of non-alcoholic steatohepatitis and cryptogenic cirrhosis following orthotopic liver transplantation in the context of the metabolic syndrome. Clin. Transplant., 26, E505-512.

Farrell, G.C. \& Larter, C.Z. (2006) Nonalcoholic fatty liver disease: from steatosis to cirrhosis. Hepatology, 43, S99-S112.

Feldstein, A.E., Charatcharoenwitthaya, P., Treeprasertsuk, S., Benson, J.T., Enders, F.B. \& Angulo, P. (2009) The natural history of non-alcoholic fatty liver disease in children: a follow-up study for up to 20 years. Gut, 58, 1538-1544.

Giorgio, V., Prono, F., Graziano, F. \& Nobili, V. (2013) Pediatric non alcoholic fatty liver disease: old and new concepts on development, progression, metabolic insight and potential treatment targets. BMC Pediatr., 13, 40.

Gonzalez-Duarte, D., Madrazo-Atutxa, A., Soto-Moreno, A. \& Leal-Cerro, A. (2012) Measurement of oxidative stress and endothelial dysfunction in patients with hypopituitarism and severe deficiency adult growth hormone deficiency. Pituitary, 15, 589-597.

Jankowska, I., Socha, P., Pawlowska, J., Teisseyre, M., Gliwicz, D.,
Czubkowski, P., Kalicinski, P., Cielecka-Kuszyk, J. \& Socha, J. (2007) Recurrence of non-alcoholic steatohepatitis after liver transplantation in a 13-yr-old boy. Pediatr. Transplant., 11, 796-798.

Jonas, M.M., Krawczuk, L.E., Kim, H.B., Lillehei, C. \& PerezAtayde, A. (2005) Rapid recurrence of nonalcoholic fatty liver disease after transplantation in a child with hypopituitarism and hepatopulmonary syndrome. Liver Transpl., 11, $108-110$

Koneru, B. \& Dikdan, G. (2002) Hepatic steatosis and liver transplantation current clinical and experimental perspectives. Transplantation, 73, 325-330.

Ludwig, J., Viggiano, T.R., McGill, D.B. \& Oh, B.J. (1980) Nonalcoholic steatohepatitis: Mayo Clinic experiences with a hitherto unnamed disease. Mayo Clin. Proc., 55, 434-438.

Nakajima, K., Hashimoto, E., Kaneda, H., Tokushige, K., Shiratori, K., Hizuka, N. \& Takano, K. (2005) Pediatric nonalcoholic steatohepatitis associated with hypopituitarism. J. Gastroenterol., 40, 312-315.

Nanda, K. (2004) Non-alcoholic steatohepatitis in children. Pediatr. Transplant., 8, 613-618.

Nishizawa, H., Iguchi, G., Murawaki, A., Fukuoka, H., Hayashi, Y., Kaji, H., Yamamoto, M., Suda, K., Takahashi, M., Seo, Y., Yano, Y., Kitazawa, R., Kitazawa, S., Koga, M., Okimura, Y., et al. (2012a) Nonalcoholic fatty liver disease in adult hypopituitary patients with GH deficiency and the impact of GH replacement therapy. Eur. J. Endocrinol., 167, 67-74.

Nishizawa, H., Takahashi, M., Fukuoka, H., Iguchi, G., Kitazawa, R. \& Takahashi, Y. (2012b) GH-independent IGF-I action is essential to prevent the development of nonalcoholic steatohepatitis in a GH-deficient rat model. Biochem. Biophys. Res. Commun., 423, 295-300.

Promrat, K., Kleiner, D.E., Niemeier, H.M., Jackvony, E., Kearns, M., Wands, J.R., Fava, J.L. \& Wing, R.R. (2010) Randomized controlled trial testing the effects of weight loss on nonalcoholic steatohepatitis. Hepatology, 51, 121-129.

Rosen, T. \& Bengtsson, B.A. (1990) Premature mortality due to cardiovascular disease in hypopituitarism. Lancet, 336, 285-288.

Schwimmer, J.B., Middleton, M.S., Deutsch, R. \& Lavine, J.E. (2005) A phase 2 clinical trial of metformin as a treatment for non-diabetic paediatric non-alcoholic steatohepatitis. Aliment. Pharmacol. Ther., 21, 871-879.

Takahashi, Y. (2012) Essential roles of growth hormone (GH) and insulin-like growth factor-I (IGF-I) in the liver. Endocr. J., 59, 955-962.

Takahashi, Y., Iida, K., Takahashi, K., Yoshioka, S., Fukuoka, H., Takeno, R., Imanaka, M., Nishizawa, H., Takahashi, M., Seo, Y., Hayashi, Y., Kondo, T., Okimura, Y., Kaji, H., Kitazawa, R., et al. (2007) Growth hormone reverses nonalcoholic steatohepatitis in a patient with adult growth hormone deficiency. Gastroenterology, 132, 938-943.

Tiniakos, D.G. (2010) Nonalcoholic fatty liver disease/nonalcoholic steatohepatitis: histological diagnostic criteria and scoring systems. Eur. J. Gastroenterol. Hepatol., 22, 643-650.

Tumgor, G. (2014) Cirrhosis and hepatopulmonary syndrome. World J. Gastroenterol., 20, 2586-2594.

Younossi, Z.M. (2008) Review article: current management of non-alcoholic fatty liver disease and non-alcoholic steatohepatitis. Aliment. Pharmacol. Ther., 28, 2-12. 